\title{
REDUCTION OF THE BEAM POINTING ERROR FOR IMPROVED FreE-SPACE OPTICAL COMMUNICATION LINK PERFORMANCE
}

\section{A PREPRINT}

\author{
๑ I. N'Doye, W. Cai, A. Alalwan, X. Sun, W. G. Headary, (๑) M.-S. Alouini, B. S. Ooi, (๑) T.-M. Laleg-Kirati * \\ Computer, Electrical and Mathematical Sciences and Engineering Division (CEMSE) \\ King Abdullah University of Science and Technology (KAUST) \\ Thuwal 23955-6900, Saudi Arabia \\ ibrahima.ndoye@kaust.edu.sa; slim.alouini@kaust.edu.sa; taousmeriem.laleg@kaust.edu.sa
}

February 10, 2021

\begin{abstract}
Free-space optical communication is emerging as a low-power, low-cost, and high data rate alternative to radio-frequency communication in short- to medium-range applications. However, it requires a close-to-line-of-sight link between the transmitter and the receiver. This paper proposes a robust $\mathcal{H}_{\infty}$ control law for free-space optical (FSO) beam pointing error systems under controlled weak turbulence conditions. The objective is to maintain the transmitter-receiver line, which means the center of the optical beam as close as possible to the center of the receiving aperture within a prescribed disturbance attenuation level. First, we derive an augmented nonlinear discrete-time model for pointing error loss due to misalignment caused by weak atmospheric turbulence. We then investigate the $\mathcal{H}_{\infty}$-norm optimization problem that guarantees the closed-loop pointing error is stable and ensures the prescribed weak disturbance attenuation. Furthermore, we evaluate the closed-loop outage probability error and bit error rate (BER) that quantify the free-space optical communication performance in fading channels. Finally, the paper concludes with a numerical simulation of the proposed approach to the FSO link's error performance.
\end{abstract}

Keywords Free-space optical (FSO) communications $\cdot \mathcal{H}_{\infty}$ pointing error control $\cdot$ Weak turbulence $\cdot$ Lognormal distribution · Linear Matrix Inequality (LMI).

\section{Introduction}

Free-space optical (FSO) communication systems have emerged as a viable technology that offers a large capacity usage (data, voice, and video) in short to medium-range applications. The range of applications include fixed-location terrestrial communication (Willebrand and Ghuman, 2001), communication between mobile robots (Kerr et al., 1996), underwater wireless optical communication (UWOC) (Oubei et al. 2015), airborne communication (Maynard, 1987), and inter-satellite communication (Chan, 2003). FSO is a line of sight communication network with a free-space or atmosphere as a channel. This channel may be turbulent, causing absorption and scattering of the optical signal due to the presence of many factors, including fog, rain, snow, and temperature variations, resulting in its deterioration (Singh and Sappal, 2019). Due to the temperature variations in the atmosphere, the refractive index changes creating Fresnel zones of different densities that scatter the laser beam from its projected path to travel diverse directions.

Atmospheric turbulence is a random phenomenon caused by the variation of temperature or humidity and the atmosphere's pressure along the propagation path. Specifically, atmospheric turbulence is highly variable and unpredictable due to weather effects (Henniger and Wilfert, 2010). It would make the optical beam fluctuated when propagating

${ }^{*}$ This work has been supported by the King Abdullah University of Science and Technology (KAUST), Base Research Fund (BAS/1/1627-01-01) to Taous Meriem Laleg. 
through the channel and finally results in misalignment due to diffraction from particulates present in the channel, resulting in enlarging the beam's size to become more significant than the receiver aperture size. Misalignment can lead to intolerable signal fades and can significantly degrade system performance. In other words, atmospheric turbulence may lead to a significant degradation in the performance of the FSO communication systems (Alheadary et al., 2015).

In addition to this, the signal propagating through the FSO channel is also perturbed by building vibrations, sways, and thermal expansions result in degradation of link performance (Shin and Chan, 2002), (Farid and Hranilovic, 2007). This misalignment can lead to pointing errors, causing the optical beam's displacement along with horizontal and vertical directions. Hence, FSO links require accurate pointing (Borah and Voelz, 2009), which means the pointing error needs to be very small to reduce the loss due to misalignment between the transmitter-receiver line.

Since FSO systems require precise pointing as the light signals are highly directional, the effect of pointing errors on link performance is a great interest for many potential applications. Several approaches have been proposed to address the LOS (Line-of-Sight) requirement in optical communication systems. In (Pontbriand et al., 2008), large-area photomultiplier tubes are used to increase the receiver's field of view. Multiple LEDs and multiple photodiodes have also been used to avoid the need for active pointing during optical-communication (Rust and Asada, 2012), (Simpson et al., 2012). However, these systems achieved the LOS through redundancy in transmitters and receivers, which resulted in a larger footprint, higher cost, and higher complexity.

Furthermore, different pointing strategies for FSO links have been proposed in (Arnon et al., 2002, Komaee et al., 2007, Yuksel et al., 2005, Liu, 2009, Cai et al., 2019). However, these methods focus on combining existing components of the pointing assembly and atmospheric turbulence effects using manual and special detection techniques. Additionally, these methods use statistical performance analysis tools to mitigate pointing error effects but did not consider the controller design aspect. Many of them did not include the influence of vibration levels and atmospheric turbulence. To alleviate these shortcomings and arrive with an accurate pointing error solution in FSO links, we propose a robust control strategy for maintaining the optical link between free-space communication stations engaged in a laser communication channel.

To the best of the authors' knowledge, designing a beam pointing error control for improved FSO link performance has not been thoroughly investigated. One of this paper's motivations is to study and characterize the lognormal turbulence fading theoretically and experimentally to construct fully auxiliary control subsystems for robust FSO links.

The contributions of this paper to the existing body in the literature in pointing error control for FSO systems are as follows.

- We propose an experimental setup and analyze the lognormal fading of the weak turbulence FSO channel.

- We derive a discrete-time nonlinear model based on a predefined autocorrelation function using the implicit Milstein scheme to simulate the lognormal optical channel state.

- Using the discrete-time model, we propose the $\mathcal{H}_{\infty}$-norm optimization problem that guarantees the closed-loop pointing error is stable and ensures a prescribed disturbance attenuation level.

- We evaluate the quality of the closed-loop pointing error control, which shows that the proposed control law can maintain the optical beam's center at the center of the receiving aperture.

- Finally, we perform numerical simulation tests of the open-loop and closed-loop outage probability error and bit error rate (BER) that quantify the free-space optical communication's performance in fading channels.

This paper is an extension of (Cai et al., 2018), with the following significant new contributions.

- A discrete-time nonlinear model based on a predefined autocorrelation function is adapted to capture the lognormal fading process and provide a comprehensive treatment of the optical beam model. Indeed, the lognormal random process is represented as a solution of a stochastic differential equation (SDE), which is approximated by a general and effective discrete-time model.

- A robust $\mathcal{H}_{\infty}$ control law is proposed to reduce the pointing error and maintain the line-of-sight link of the optical beam under controlled lognormal weak-turbulence conditions.

- The communication performance metrics using the outage probability error and the bit error rate (BER) are evaluated and analyzed.

The outline of the paper is organized as follows: In Section 2 we describe the experimental setup of the FSO channel in which we characterize the lognormal fading and derive the discrete-time lognormal optical channel state. In Section 3 . we formulate the pointing error problem to maintain the centroid of the optical beam as close as possible to the center of the photodetector. In Section 4 , the main results of the pointing error problem based on the $\mathcal{H}_{\infty}$-norm optimization 


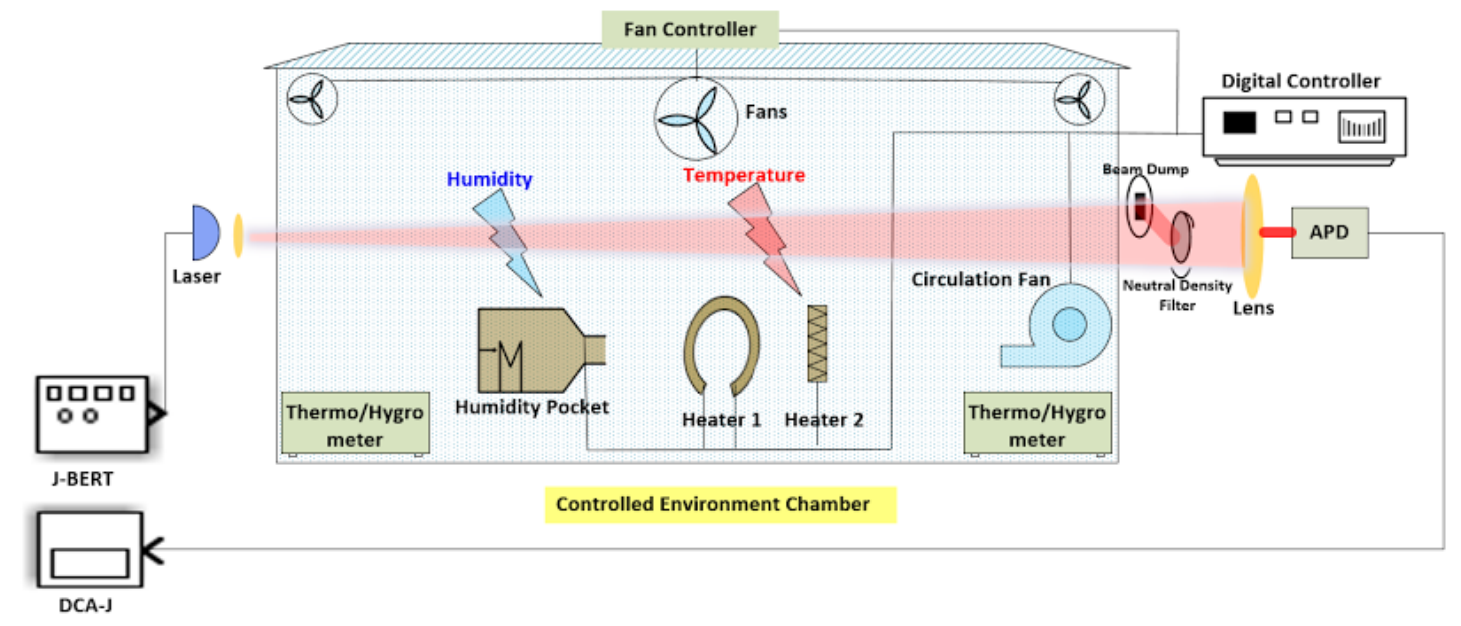

Figure 1: Block diagram of the experiment FSO setup.

method under controlled weak-turbulence conditions are derived. In Section 5 , we evaluate the quality of the pointing error control and the communication performance metrics through numerical simulation tests. Finally, concluding remarks of the proposed robust pointing error control are presented in Section 6 .

Notations. $M^{T}$ is the transpose of $M$. In symmetric block matrices, the symbol $(\star)$ in any matrix represents for any element that is induced by transposition. $\|$.$\| is the induced 2-norm. \mathbf{0}$ and $\mathbf{I}$ stand for the null matrix and the identity matrix of appropriate dimensions, respectively.

\section{Experimental Setup and Dynamic Model for Weak Turbulence FSO Channel}

The FSO link consists of a transmitter and receiver separated by the atmospheric channel. Here we set up an experimental system, the schematic diagram of the experimental line-of-sight FSO link is shown in Fig. 11 The optical signal amplitude through the FSO channel is fluctuated due to the atmospheric turbulence. Many statistical models of the intensity fluctuation through FSO channels have been proposed in the literature for distinct turbulence regimes. For weak turbulence conditions, the most widely used model is the log-normal distribution, which has been validated through studies (Farid and Hranilovic, 2007), (Majumdar and Ricklin. 2008), (Zhu and Kahn, 2002). It is a well-known modeling approach and has been adopted in many calculations for the turbulence channel. This paper will focus on the weak turbulence; therefore, the lognormal model will be used throughout.

\subsection{Lognormal Weak Turbulence Characterization}

The laboratory atmospheric channel is a closed glass chamber with a dimension of $100 \times 35 \times 42 \mathrm{~cm}^{3}$ as depicted in Fig. 2 with the aim of observing the effect of atmospheric turbulence on the laser beam propagating through the channel. The main parameters of the FSO link are given in Table 1. The probability density function (PDF) of the received irradiance $I$ due to the turbulence is derived by (Osche, 2002), (Ghassemlooy et al., 2012a)

$$
p(I)=\frac{1}{\sqrt{2 \pi \sigma^{2}}} \frac{1}{I} \exp \left\{-\frac{\left(\ln \left(I / I_{0}\right)+\sigma^{2} / 2\right)^{2}}{2 \sigma^{2}}\right\}
$$

where $I_{0}$ is the irradiance when there is no turbulence and $\sigma^{2}$ is the log-amplitude variance or scintillation index in the channel.

The measured eye-diagrams for the received signal are depicted in Fig. 3 without turbulence and in Figs. 4 and 5 with weak turbulence. We observe that the eye-opening is smaller in the presence of turbulence, which results in a considerable level of signal intensity fluctuation and also reduces the FSO performance link.

Fig. 7 shows the histogram and the curve fitting plots of the received intensity signal without turbulence. As we can see, the PDF distribution is nearly Gaussian for lower values of $\sigma^{2}$. The experimental snapshot intensity sensed by the PD can be fitted with a nearly Gaussian distribution, as illustrated in Fig. 6

Figs. 8 and 9 illustrate the histograms and the curve fitting plots of the received intensity signal with turbulence. It is clear that the PDF has a good fitting with the lognormal distribution, and the estimated scintillation index $\sigma^{2}$ falls within 


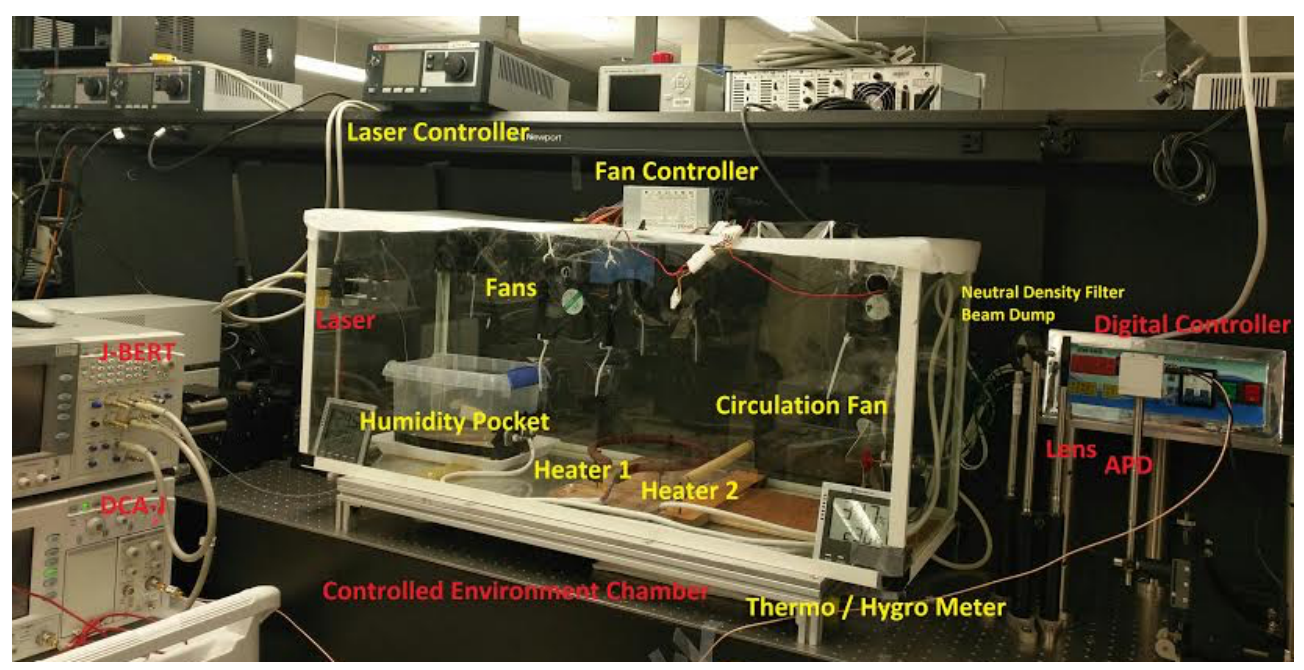

Figure 2: Experimental laboratory turbulence chamber.

Table 1: Parameters of the FSO link.

\begin{tabular}{|c|c|c|}
\hline Description & Parameter & Value \\
\hline Data & $\begin{array}{c}\text { Format } \\
\text { PRBS length } \\
\text { Signal intensity } V_{\text {peak-to-peak }} \\
\text { Data rate }\end{array}$ & $\begin{array}{c}\text { OOK NRZ } \\
2^{10}-1 \\
1.78 \mathrm{~V} \\
622.082 \mathrm{Mb} / \mathrm{s}\end{array}$ \\
\hline Laser diode & \begin{tabular}{|c|} 
Type \\
Peak wavelength \\
Optical Output Power \\
Operating current/voltage
\end{tabular} & $\begin{array}{c}\text { LP642-SF20 } \\
642 \mathrm{~nm} \\
20 \mathrm{~mW} \\
0.089 \mathrm{~A} / 2.371 \mathrm{~V}\end{array}$ \\
\hline Photodetector & $\begin{array}{c}\text { Type } \\
\text { Spectral range } \\
\text { Maximum gain } \\
\text { Detector diameter } \\
\text { Rise time }\end{array}$ & $\begin{array}{c}\text { APD210 } \\
400-1000 \mathrm{~nm} \\
2.5 \times 10^{5} \mathrm{~V} / \mathrm{W} \\
0.5 \mathrm{~mm} \\
0.5 \mathrm{~ns}\end{array}$ \\
\hline Lens & $\begin{array}{c}\text { Type } \\
\text { Diameter } \\
\text { Focal length }\end{array}$ & $\begin{array}{c}\text { LA1417-A } \\
50.8 \mathrm{~mm} \\
150 \mathrm{~mm}\end{array}$ \\
\hline Transmitter & Type & N4903B J-BERT \\
\hline Receiver & $\begin{array}{c}\text { Type } \\
\text { Sampling time }\end{array}$ & $\begin{array}{c}86100 \mathrm{C}-\mathrm{DCA}-\mathrm{J} \\
0.2 \mathrm{~ns}\end{array}$ \\
\hline Chamber & Dimension & $100 \times 35 \times 42 \mathrm{~cm}^{3}$ \\
\hline
\end{tabular}

the range of $[0,0.1]$ is characterized by weak turbulence regime (Ghassemlooy et al. 2012a). The results also show the theoretical red-dotted-lines fit well with the simulation solid-blue-lines, which demonstrate the close resemblance between the PDFs fading statistics based on lognormal distributions of the theoretical predefined autocorrelation function (see (Primak et al., 2005; Kontorovich and Lyandres, 1995; Bykhovsky et al., 2015; Neuenkirch and Szpruch. 2014) and the simulation for short-range turbulent-channel communication experiment. As $\sigma^{2}$ increases, the distribution is more skewed with a long tail toward the infinity and reduced peak of probability density as a result of signal fading. 


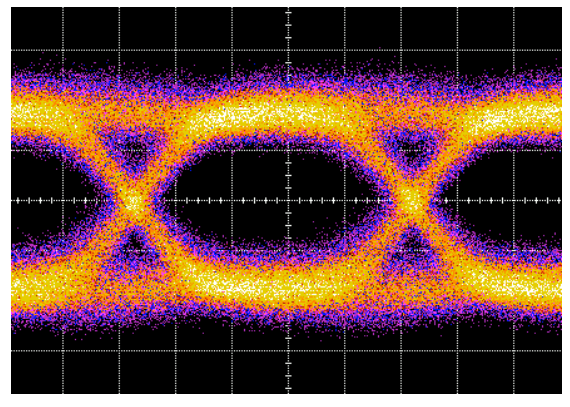

Figure 3: Measured screen shot eye-diagram of received intensity signal without turbulence

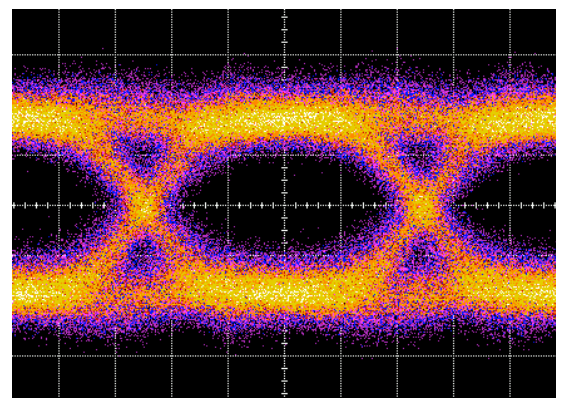

Figure 4: Measured screen shot eye-diagram of received intensity signal under weak turbulence: $\sigma^{2}=0.0380$.

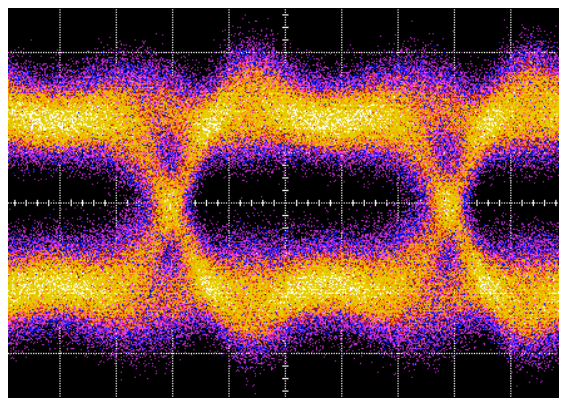

Figure 5: Measured screen shot eye-diagram of received intensity signal under weak turbulence: $\sigma^{2}=0.0576$.

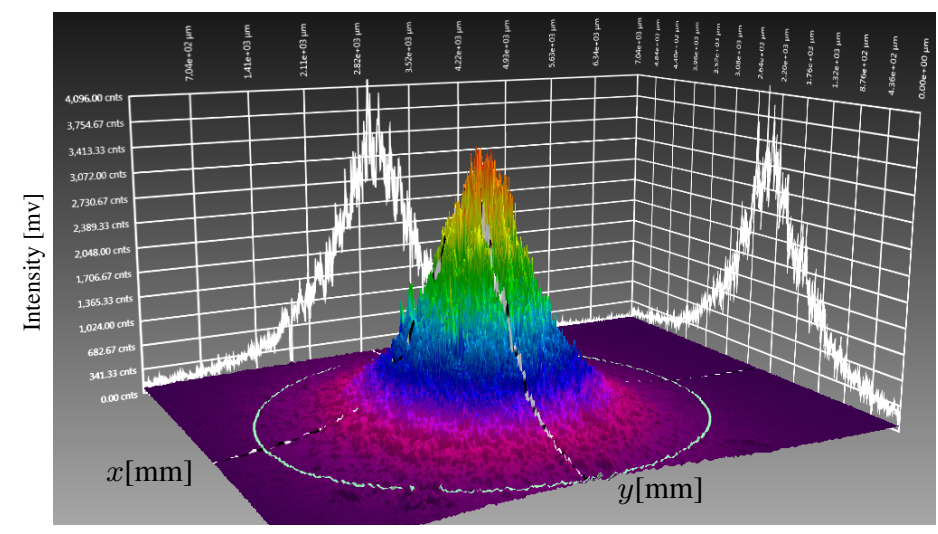

Figure 6: Experimental snapshot intensity of the laser beam obeying to Gaussian distribution.

\subsection{Discrete-Time Lognormal Optical Channel State}

The most important property of the optical beam is the PDF of gain samples, so we use a modeling approach to get the optical beam position. The modeling approach is based on 1D lognormal distributed samples with a corresponding correlation function, as illustrated in Figs. 7, 8 and 9 . The first step is to efficiently approximate the weak turbulence level of the FSO chamber, which means to emulate the variance of the atmospheric turbulence. We verify the theoretical results of the lognormal process with the predefined autocorrelation function using the implicit Milstein scheme for the channel states, which converges to a simple discrete-time differential equation (Neuenkirch and Szpruch. 2014), (Bykhovsky, 2015). The implicit discrete-time Milstein scheme for the lognormal distribution describing the simulated lognormal optical beam channel state and its relative position is generated by the following nonlinear discrete-time 


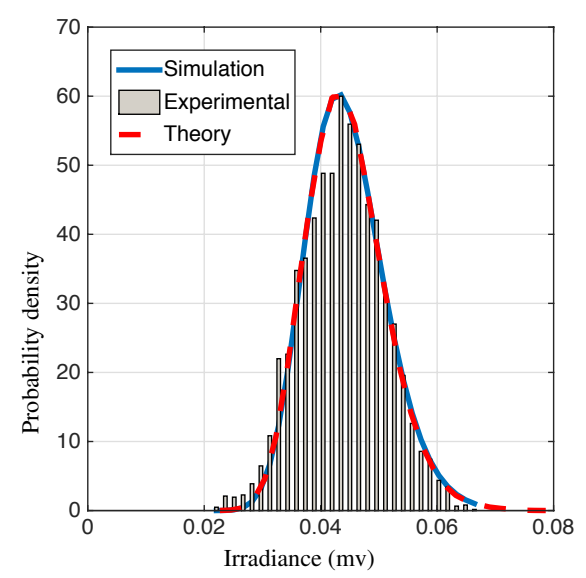

Figure 7: Gaussian PDF received distribution without turbulence (the curve fitting is shown by solid lines)

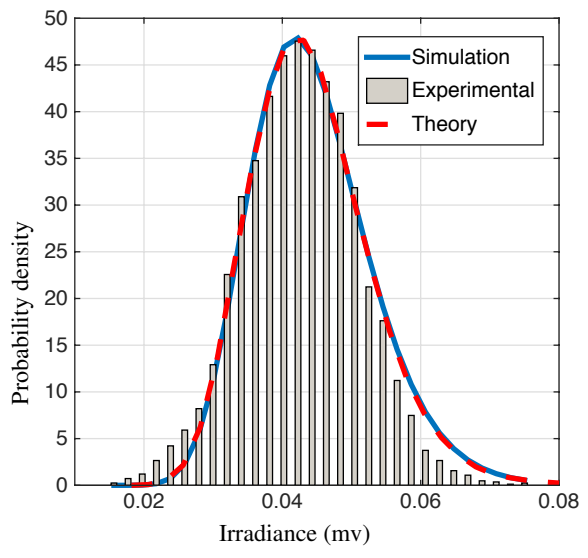

Figure 8: Log-normal PDF received distribution under weak turbulence: $\sigma^{2}=0.0380$ (the curve fitting is shown by solid lines).

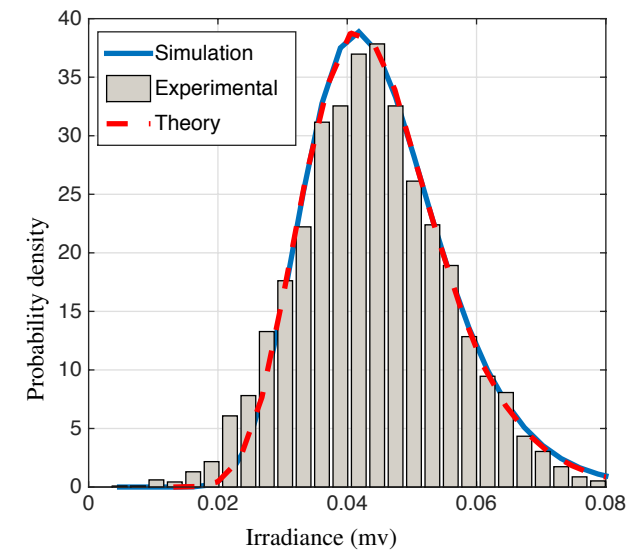

Figure 9: Log-normal PDF received distribution under weak turbulence: $\sigma^{2}=0.0576$ (the curve fitting is shown by solid lines).

state-space equations (Neuenkirch and Szpruch, 2014), (Kontorovich and Lyandres, 1995), (Bykhovsky, 2015)

$$
\left\{\begin{array}{l}
x_{k+1}^{p}=a^{p} x_{k}^{p}+\varphi\left(x_{k}^{p}\right)+b^{p} u_{k}^{p}+r^{p} w_{k}^{p} \\
\theta_{k}=c^{p} x_{k}^{p}
\end{array}\right.
$$

where

$K$ is given by

$$
\varphi\left(x_{k}^{p}\right)=-\frac{K}{2 \sigma^{2} x_{k}^{p}}\left[\ln \left(x_{k}^{p} / I_{0}\right)\right], \quad r^{p}=\sqrt{K \times \Delta t},
$$

$$
K=\frac{2 I_{0}^{2} \exp \left(\sigma^{2}\right)\left[\exp \left(\sigma^{2}\right)-1\right]}{\tau_{c}},
$$

where $k \in \mathbb{Z}^{+}$is the set of all nonnegative integers, $x_{k}^{p} \in \mathbb{R}$ is the simulated optical channel state which can be considered as a moving object, $\theta_{k} \in \mathbb{R}$ is the position of the optical beam transmitter, $w_{k}^{p} \in \mathbb{R}$ are samples of the white Gaussian noise and $\tau_{c}$ is a predefined correlation time, $u_{k}^{p}$ is the bounded control input through which the optical channel state and transmission angle are changed. $\Delta t$ is the sampling time, $a^{p} \in \mathbb{R}, b^{p} \in \mathbb{R}, c^{p} \in \mathbb{R}$ and $r^{p} \in \mathbb{R}$ are constant values. The nonlinearity $\varphi\left(x_{k}^{p}\right) \in \mathcal{P} \subseteq \mathbb{R}^{+}$represents here the full signal strength model. It is differentiable with $\varphi(0)=0$, locally Lipschitz and also monotonically increasing in $\mathcal{P}$. The time-correlated position of the transmitted optical beam that was generated by (2) with $\sigma^{2}=0.0380, \tau_{c}=0.1, a^{p}=1, b^{p}=1$ and $c^{p}=1$ is illustrated in Fig. 10 


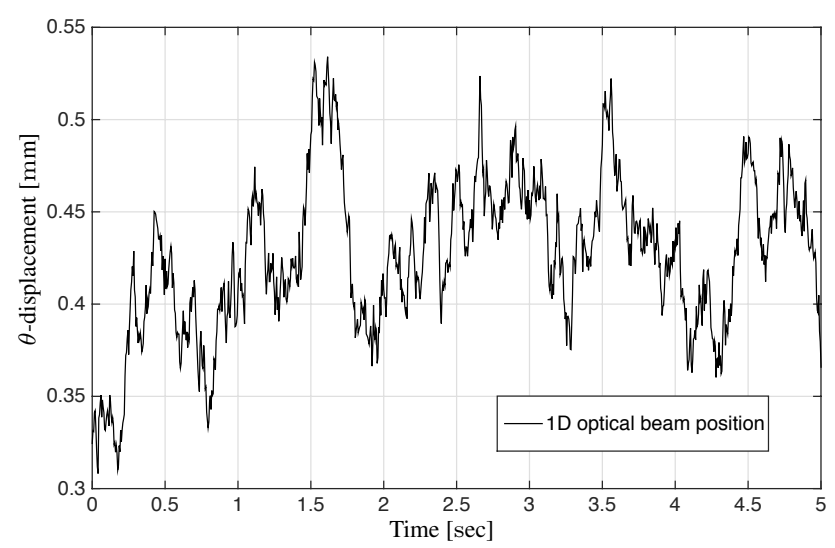

Figure 10: Position of the transmitted optical beam motion versus time.

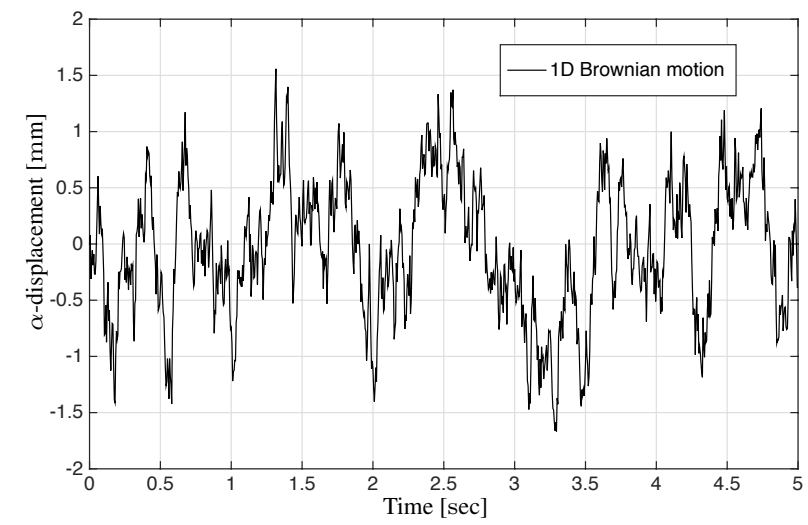

Figure 11: Position of the 1D receiving aperture motion versus time.

\subsection{Receiving Aperture Model}

Although the photodetector's receiver aperture is fixed, it still suffers some random physical vibrations due to thermal expansion, voltage jitter, etc (see (Cai et al., 2019)). So, the receiving aperture motion is assumed similar to the Brownian motion of a particle subjected to excitation, as showed in Fig. 111. The Brownian motion is given by the generalized differential equation (Volpe and Volpe, 2013; Amari et al., 2013, 2014)

$$
m \frac{\mathrm{d}^{2} x(t)}{\mathrm{d} t^{2}}=-\gamma_{1} \frac{\mathrm{d} x(t)}{\mathrm{d} t}-k_{1} \frac{\mathrm{d} x(t)}{\mathrm{d} t}+\sqrt{2 k_{B} T \gamma_{1}} W(t),
$$

where $x(t)$ is the trajectory of the particle with respect to the center, $m$ is the particle mass, $\gamma_{1}$ is the friction exerted by the surrounding medium on the particle, $k$ is the optical trap stiffness, $k_{B} T$ is the thermal energy unit, $k_{B}$ is the Boltzmann constant, $T$ is the absolute temperature and $W(t)$ is white Gaussian noise. The discrete-time state space of the receiving aperture model is derived from the discretized particle Brownian motion (5). It is given as follows (Volpe and Volpe, 2013; Amari et al. 2013, 2014)

$$
\left\{\begin{array}{l}
x_{k+1}^{l}=a^{l} x_{k}^{l}+r^{l} w_{k}^{l}, \\
\alpha_{k}=c^{l} x_{k}^{l},
\end{array}\right.
$$

where $x_{k}^{l}$ is the source position, $\alpha_{k}$ is the measured position of the receiving aperture motion, $w_{k}^{l}$ is a standard white Gaussian noise, $\Delta T$ is the discretization time step, $a^{l}=\left(1-\frac{k_{1} \Delta T}{\gamma_{1}}\right), r^{l}=\sqrt{2 k_{B} T \gamma_{1}}$ and $c^{l}=1$.

\section{Problem Formulation}

We consider a one-way optical link that consists of an optical transmitter and an optical receiver. Both are subject to relative motions. The emitted optical beam has a non-uniform intensity profile, which is assumed to be Gaussian 
(Gagliardi and Karp, 1995) and can be considered as a moving object. The goal is to control how the position of the object change in time. The receiver's aperture is assumed to be smaller than the received optical beam so that the receiver can collect only a fraction of the optical beam (Komaee et al., 2007). This captured fraction can be enlarged by active pointing whose objective is to maintain the optical beam's center at the center of the receiving aperture. A photodetector is used at the receiver to measure the optical beam's intensity profile that strikes its aperture (Komaee et al. 2007). The output is then sent as feedback through an optical link or low-bandwidth RF channel and used to adjust the transmitter's position. Fig. 12 illustrates the block diagram of this active pointing scheme under weak controlled turbulence.

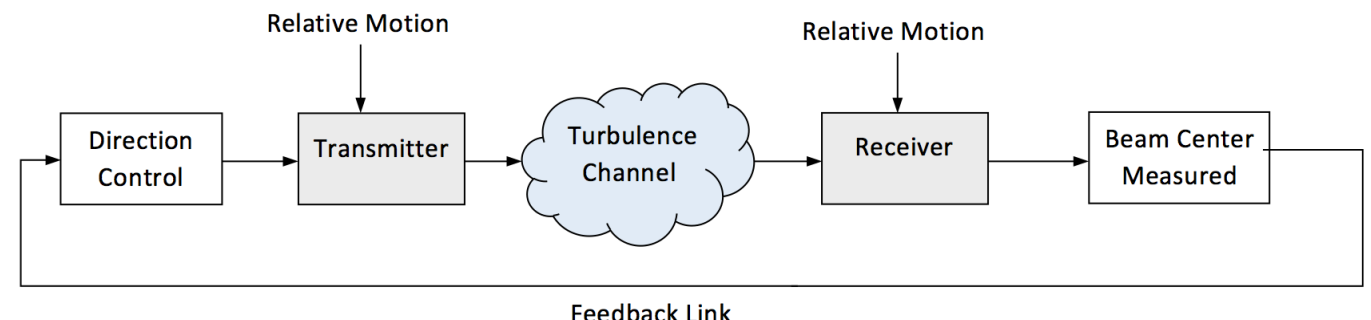

Figure 12: Active pointing scheme for a short range free-space optical channel.

The discrete-time model considered in this study has been derived from the model structure that was introduced for the stochastic state-space model (Komaee et al. 2007), (Bykhovsky, 2015). Indeed, the lognormal random process is represented as a solution of a stochastic differential equation (SDE), which is approximated by a general and effective discrete-time model. The model mainly describes the relative position's effect between the transmitter and the receiver on the signal strength. We denote the transmitted optical beam position to a fixed coordinate system by the vector $\theta_{k}$ and the position of the receiving aperture of the stations to the same coordinate system by $\alpha_{k}$. We assume that the receiving aperture is held perpendicular to the line-of-sight optical beam. The relative displacement of the optical beam center to the receiving aperture center is given by $y_{k}=d\left(\theta_{k}-\alpha_{k}\right)$ where $d$ is the distance between the transmitter and receiver. Fig. 13 illustrates the optical beam in the plane of the receiving aperture and the displacement vector $y_{k}$.

The pointing error $y_{k}=d\left(\theta_{k}-\alpha_{k}\right)$ is a linear function of $x_{k}^{p}$ and $x_{k}^{l}$. It can be written as the following augmented system form

where

$$
\left\{\begin{array}{l}
x_{k+1}=\mathbb{A} x_{k}+\varphi\left(x_{k}\right)+\mathbb{B} u_{k}+\mathbb{R} w_{k} \\
\epsilon_{k}=\mathbb{C} x_{k}
\end{array}\right.
$$

$$
\mathbb{A}=\left[\begin{array}{cc}
a^{p} & \mathbf{0} \\
\mathbf{0} & a^{l}
\end{array}\right], \mathbb{B}=\left[\begin{array}{c}
b^{p} \\
\mathbf{0}
\end{array}\right], \mathbb{R}=\left[\begin{array}{l}
r^{p} \\
r^{l}
\end{array}\right], \mathbb{C}=\left[\begin{array}{ll}
r^{p} & -r^{l}
\end{array}\right],
$$

and $x_{k}=\left[\begin{array}{l}x_{k}^{p} \\ x_{k}^{l}\end{array}\right] \in \mathbb{R}^{2}$ is the augmented state vector, $w_{k}=\left[\begin{array}{ll}w_{k}^{p} & w_{k}^{l}\end{array}\right]$ is the augmented disturbance vector, $y_{k}=d \epsilon_{k}$ is the pointing error and $\epsilon_{k}=\theta_{k}-\alpha_{k}$.

Since $\varphi\left(x_{k}^{p}\right)$ is Lipschitz, then the augmented nonlinearity $\varphi\left(x_{k}\right)$ is assumed to satisfy the following bound

where $\mathbb{H}$ is a constant matrix.

$$
\varphi\left(x_{k}\right)^{T} \varphi\left(x_{k}\right) \leqslant x_{k}^{T} \mathbb{H}^{T} \mathbb{H} x_{k},
$$

The robust control problem studied in this paper consists in minimizing the closed-loop pointing error while ensuring the disturbance attenuation level. The objective is to maintain the centroid of the optical beam as close as possible to the center of the photodetector. This pointing problem can be interpreted as finding a set-point $u_{k}=-\mathbb{K} x_{k}$ depending on $x_{k}$ such that the following $\mathcal{H}_{\infty}$ norm of the pointing error $y_{k}$ with respect to disturbance $w_{k}$ is satisfied i.e:

$$
\left\|y_{k}\right\| \leqslant \varepsilon\left\|w_{k}\right\| \text {, }
$$

with $\varepsilon$ being the smallest positive real to be minimized.

\section{Robust $\mathcal{H}_{\infty}$ Pointing Error Control for FSO}

In this section, we consider the $\mathcal{H}_{\infty}$-norm optimization problem that guarantees the closed-loop pointing error $y_{k}$ is stable and ensures the disturbance attenuation level $\left\|y_{k}\right\| \leqslant \varepsilon\left\|w_{k}\right\|$ for a prescribed attenuation level $\varepsilon>0$. 


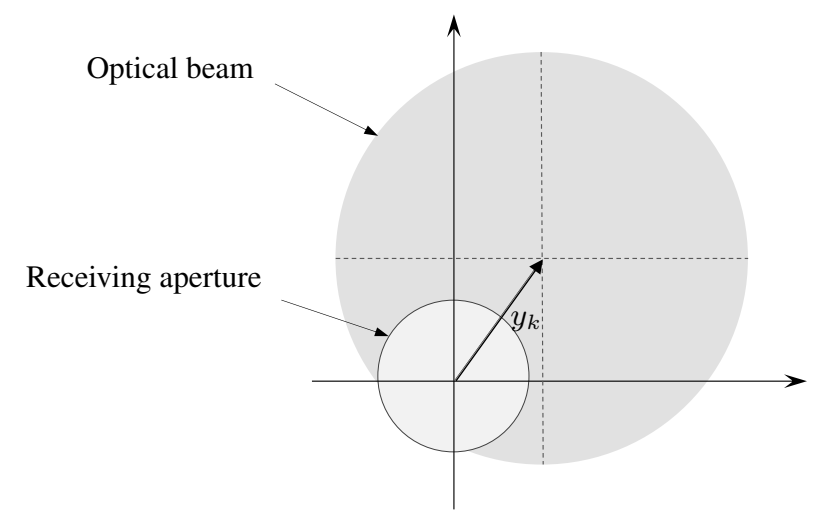

Figure 13: Optical beam, receiving aperture and the displacement vector $y_{k}$.

The following theorem provides the stability and the absolute pointing error of the augmented system (7).

Theorem 1 If there exist matrices $\mathbb{Y}=\mathbb{Y}^{T}>0, \mathbb{S}$ and scalar $\varepsilon$ such that the following LMI condition

$$
\begin{gathered}
\text { } \min _{\mathbb{S}, \mathbb{Y}>0} \varepsilon \text { subject to } \\
{\left[\begin{array}{cccccc}
-\mathbb{Y} & \mathbf{0} & \mathbf{0} & \mathbb{Y} \mathbb{A}^{T}-\mathbb{S}^{T} \mathbb{B} & \mathbb{Y} \mathbb{C}^{T} & \mathbb{Y} \mathbb{H}^{T} \\
(\star) & -\varepsilon^{2} \mathbf{I} & \mathbf{0} & \mathbb{R}^{T} & \mathbf{0} & \mathbf{0} \\
(\star) & (\star) & -\delta \mathbf{I} & \mathbb{Y} & \mathbf{0} & \mathbf{0} \\
(\star) & (\star) & (\star) & -\mathbb{Y} & \mathbf{0} & \mathbf{0} \\
(\star) & (\star) & (\star) & (\star) & -\mathbf{I} & \mathbf{0} \\
(\star) & (\star) & (\star) & (\star) & (\star) & -\delta^{-1} \mathbf{I}
\end{array}\right]<0 .}
\end{gathered}
$$

has a feasible solution with $\mathbb{K}=\mathbb{S Y} \mathbb{Y}^{-1}$, then

i) the augmented closed-loop error system (7) is stable for $w_{k}=0$.

ii) and the pointing error $y_{k}$ satisfies the disturbance attenuation condition for $w_{k} \neq 0$ and a specific attenuation factor $\varepsilon>0$,

$$
\left\|y_{k}\right\| \leqslant \varepsilon\left\|w_{k}\right\| .
$$

Proof 1 Let us define the Lyapunov function $V_{k}=x_{k}^{T} \mathbb{P} x_{k}$ with $\mathbb{P}=\mathbb{P}^{T}>0$ and the $\mathcal{H}_{\infty}$ cost (Boyd et al. . 1994) in equation (11) as follows

$$
J \triangleq \sum_{k=0}^{N-1}\left(y_{k}^{T} y_{k}-\varepsilon^{2} w_{k}^{T} w_{k}\right)
$$

over the time interval $[0, N-1], N \in \mathbb{N}$. If $V_{0}=0$, i.e, all the initial conditions are null then, inequality (11) holds if the following satisfies along the trajectories of system (7)

$$
J<\sum_{k=0}^{N-1}\left(x_{k}^{T} \mathbb{C}^{T} \mathbb{C} x_{k}-\varepsilon^{2} w_{k}^{T} w_{k}+V_{k+1}-V_{k}\right) .
$$

A sufficient condition to fulfill the inequality (13) is to guarantee for all $k \in \mathbb{Z}_{\geqslant 0}$

$$
x_{k}^{T} \mathbb{C}^{T} \mathbb{C} x_{k}-\varepsilon^{2} w_{k}^{T} w_{k}+V_{k+1}-V_{k}<0 .
$$

Inequality (14) can be written as follows

$$
\begin{aligned}
& x_{k}^{T}\left[(\mathbb{A}-\mathbb{B} \mathbb{K})^{T} \mathbb{P}(\mathbb{A}-\mathbb{B} \mathbb{K})-\mathbb{P}\right] x_{k}+x_{k}^{T}(\mathbb{A}-\mathbb{B} \mathbb{K})^{T} \mathbb{P} \mathbb{R} w_{k}+x_{k}^{T}(\mathbb{A}-\mathbb{B} \mathbb{K})^{T} \mathbb{P} \varphi\left(x_{k}\right)+w_{k}^{T} \mathbb{R}^{T} \mathbb{P}(\mathbb{A}-\mathbb{B} \mathbb{K}) x_{k} \\
& +w_{k}^{T} \mathbb{R}^{T} \mathbb{P} \mathbb{R} w_{k}+\varphi^{T}\left(x_{k}\right) \mathbb{P}(\mathbb{A}-\mathbb{B} \mathbb{K}) x_{k}+\varphi^{T}\left(x_{k}\right) \mathbb{P} \varphi\left(x_{k}\right)+\varphi^{T}\left(x_{k}\right) \mathbb{P} \mathbb{R} w_{k}+w_{k}^{T} \mathbb{R}^{T} \mathbb{P} \varphi\left(x_{k}\right)+x_{k}^{T} \mathbb{C}^{T} \mathbb{C} x_{k}-\varepsilon^{2} w_{k}^{T} w_{k}<0 .
\end{aligned}
$$




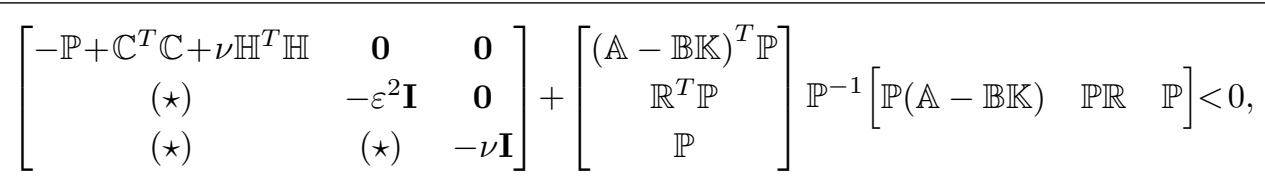

Inequality 15 can be expressed as

$$
\left[\begin{array}{c}
x_{k} \\
w_{k} \\
\varphi\left(x_{k}\right)
\end{array}\right]^{T}\left[\begin{array}{ccc}
\Xi & (\mathbb{A}-\mathbb{B} \mathbb{K})^{T} \mathbb{P} \mathbb{R} & (\mathbb{A}-\mathbb{B} \mathbb{K})^{T} \mathbb{P} \\
(\star) & -\varepsilon^{2} \mathbf{I}+\mathbb{R}^{T} \mathbb{P} R & \mathbb{R}^{T} \mathbb{P} \\
(\star) & (\star) & \mathbb{P}
\end{array}\right]\left[\begin{array}{c}
x_{k} \\
w_{k} \\
\varphi\left(x_{k}\right)
\end{array}\right]<0
$$

where $\Xi=-\mathbb{P}+(\mathbb{A}-\mathbb{B} \mathbb{K})^{T} \mathbb{P}(\mathbb{A}-\mathbb{B} \mathbb{K})+\mathbb{C}^{T} \mathbb{C}$.

Using the fact that constraint (8) is equivalent to the following quadratic inequality for any $\delta>0$

$$
\delta\left[\begin{array}{c}
x_{k} \\
w_{k} \\
\varphi\left(x_{k}\right)
\end{array}\right]^{T}\left[\begin{array}{ccc}
\mathbb{H}^{T} \mathbb{H} & \mathbf{0} & \mathbf{0} \\
(\star) & \mathbf{0} & \mathbf{0} \\
(\star) & (\star) & -\mathbf{I}
\end{array}\right]\left[\begin{array}{c}
x_{k} \\
w_{k} \\
\varphi\left(x_{k}\right)
\end{array}\right] \geqslant 0
$$

Combining together (16) and (17) gives

$$
\left[\begin{array}{c}
x_{k} \\
w_{k} \\
\varphi\left(x_{k}\right)
\end{array}\right]^{T}\left[\begin{array}{ccc}
\Xi_{1} & (\mathbb{A}-\mathbb{B} \mathbb{K})^{T} \mathbb{P R} & (\mathbb{A}-\mathbb{B} \mathbb{K})^{T} \mathbb{P} \\
(\star) & -\varepsilon^{2} \mathbf{I}+\mathbb{R}^{T} \mathbb{P} \mathbb{R} & \mathbb{R}^{T} \mathbb{P} \\
(\star) & (\star) & \mathbb{P}-\delta \mathbf{I}
\end{array}\right]\left[\begin{array}{c}
x_{k} \\
w_{k} \\
\varphi\left(x_{k}\right)
\end{array}\right]<0,
$$

where $\Xi_{1}=-\mathbb{P}+(\mathbb{A}-\mathbb{B} \mathbb{K})^{T} \mathbb{P}(\mathbb{A}-\mathbb{B} \mathbb{K})+\mathbb{C}^{T} \mathbb{C}+\delta \mathbb{H}^{T} \mathbb{H}$.

Using the well-known Schur complement, we obtain inequality (19).

Applying again the Schur complement to (19), then we obtain the following sufficient condition

$$
\left[\begin{array}{cccccc}
-\mathbb{P} & \mathbf{0} & \mathbf{0} & (\mathbb{A}-\mathbb{B} \mathbb{K})^{T} \mathbb{P} & \mathbb{C}^{T} & \mathbb{H}^{T} \\
(\star) & -\varepsilon^{2} \mathbf{I} & \mathbf{0} & \mathbb{R}^{T} \mathbb{P} & \mathbf{0} & \mathbf{0} \\
(\star) & (\star) & -\delta \mathbf{I} & \mathbb{P} & \mathbf{0} & \mathbf{0} \\
(\star) & (\star) & (\star) & -\mathbb{P} & \mathbf{0} & \mathbf{0} \\
(\star) & (\star) & (\star) & (\star) & -\mathbf{I} & \mathbf{0} \\
(\star) & (\star) & (\star) & (\star) & (\star) & -\delta^{-1} \mathbf{I}
\end{array}\right]<0 .
$$

Pre- and post-multiplying inequality $(20)$ by

$\operatorname{diag}[\mathbb{Y}, \mathbf{I}, \mathbf{I}, \mathbb{Y}, \mathbf{I}, \mathbf{I}]$ and using $\mathbb{Y}=\mathbb{P}^{-1}$ and $\mathbb{S}=\mathbb{K} \mathbb{Y}$ which yields to the LMI (10).

The fulfillment of inequality (10) implies the fulfillment of the optimality condition:

$$
\left\|y_{k}\right\| \leqslant \varepsilon\left\|w_{k}\right\|, \quad u_{k} \neq 0, w_{k} \neq 0 .
$$

This completes the proof.

\section{Numerical simulations}

This section evaluates both the quality of the pointing error performance and the communication performance metrics to ensure accurate pointing and improve the optical communication link performance.

\subsection{Pointing Error Performance}

To evaluate the quality of the pointing error obtained by the LMI condition given in (10). The YALMIP interface (Lofberg, 2016) to MATLAB 8.5 with SDPT3 optimization toolbox (Toh et al., 1999) was used to provide solutions. 


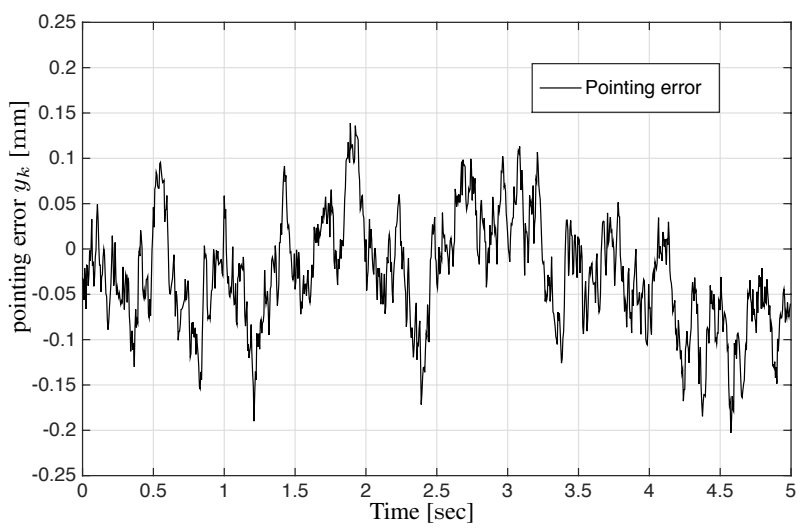

Figure 14: Closed-loop pointing error versus Time.

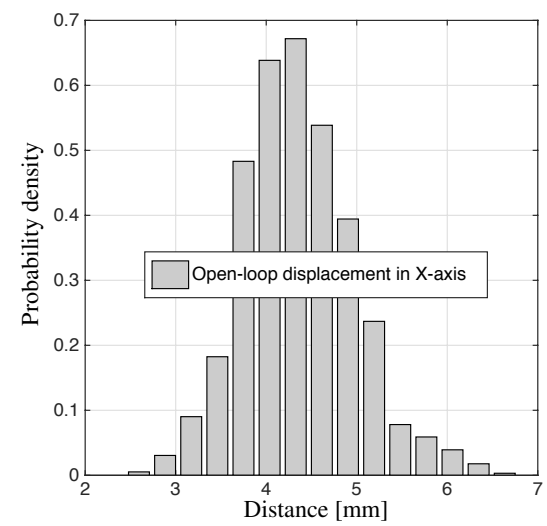

Figure 15: Open-loop output-error displacement.

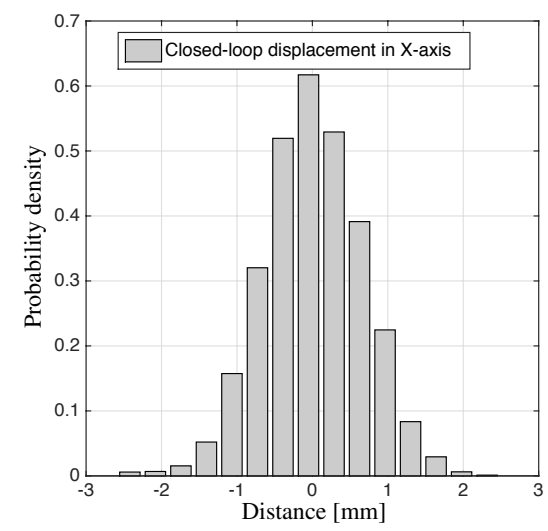

Figure 16: Closed-loop output-error displacement.

The performance of the pointing error control strategy is visualized through Fig. 14 where $\varepsilon=0.32$ and $\mathbb{K}=$ $\left[\begin{array}{ll}0.275-0.019\end{array}\right]$. We observe clearly that the closed-loop pointing maintains a small alignment error between the optical beam transmitter and the receiving aperture system with a relatively error amplitude less than $\pm 0.2 \mathrm{~mm}$ from the detector diameter, which is $\pm 0.5 \mathrm{~mm}$. Therefore the maximum relative error is $\pm 40 \%$.

Statistical simulated values provide more analytical insight. Figs. 15 and 16 depict histogram data of the open-loop and closed-loop output-error displacements, respectively. The open-loop error displacement varies from $+2 \mathrm{~mm}$ to $+7 \mathrm{~mm}$. The performance of open-loop with an output-error variance $\sigma^{2}=0.0380$ as expected due to bias and drift terms is inadequate and do not satisfactorily stabilize the beam at the center. For the closed-loop output-error feedback, the beam is stabilized at the center of the sensing device, the closed-loop error displacement varies from $-3 \mathrm{~mm}$ to $+3 \mathrm{~mm}$ and the output-error variance is $\sigma^{2}=0.0231$ which is $\pm 39 \%$ reduced from the open-loop output error. 


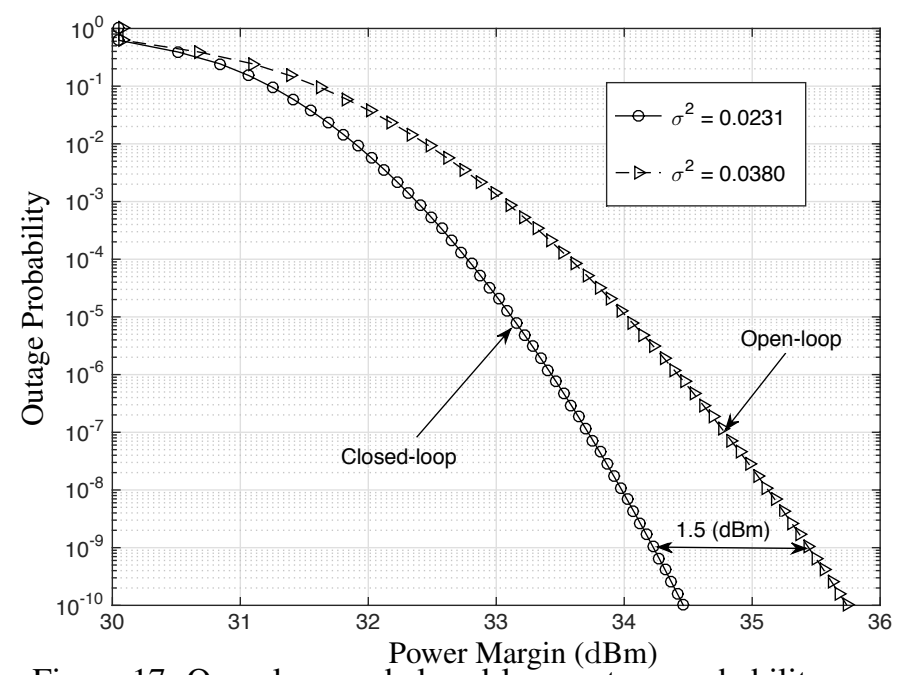

Figure 17: Open-loop and closed-loop outage probability errors.

\subsection{Communication Performance Metrics}

The outage probability error and bit error rate (BER) are metrics for quantifying communication systems' performance in fading channels. FSO system with a good average BER can temporarily suffer from increases in pointing error rate due to fading effects (Farid and Hranilovic, 2007, Alouini and Simon, 2002, Ghassemlooy et al., 2012b; Yang et al., 2014). The outage probability is given as follows (Ghassemlooy et al. 2012b)

$$
P_{o}(I)=\int_{0}^{I_{0} / m} \frac{1}{\sqrt{2 \pi \sigma^{2}}} \frac{1}{I} \exp \left\{-\frac{\left(\ln \left(I / I_{0}\right)+\sigma^{2} / 2\right)^{2}}{2 \sigma^{2}}\right\} \mathrm{d} I,
$$

where $m$ is the power margin which is introduced to account for the extra power needed to cater for turbulence-induced signal fading. Using Chernoff upper bound on (22), an approximate power margin, $m$, needed to obtain $P_{o}$ can be obtained as follows (Ghassemlooy et al. 2012b)

$$
m \approx \exp \left(\sqrt{-2 \ln 2 P_{o} \sigma^{2}}+\sigma^{2} / 2\right) .
$$

The evaluation of this outage probability error for the FSO link in open-loop and closed-loop conditions is depicted in Fig. 17. The closed-loop outage probability error which output-error variance $\sigma^{2}=0.0231$ is reduced of $1.5 \mathrm{dBm}$ from the open-loop outage probability error which output-error variance $\sigma^{2}=0.0380$. For example, to achieve an outage probability of $10^{-6}$ about $34.3 \mathrm{dBm}$ of extra power is needed in open-loop condition at $\sigma^{2}=0.0380$. This is reduced to $33.2 \mathrm{dBm}$ in closed-loop condition as the scintillation strength decreases to $\sigma^{2}=0.0231$.

The average BER of OOK-based FSO in atmospheric turbulence is defined as (Yang et al., 2014)

$$
\mathrm{BER}=\int_{0}^{\infty} p(I) Q\left(\frac{\eta I}{\sqrt{2 N_{0}}}\right) \mathrm{d} I,
$$

where $Q(x)=\int_{x}^{+\infty} \exp \left(-t^{2} / 2\right) \mathrm{d} t, \eta$ is the optical-to-electrical conversion coefficient, $I$ represents the received optical intensity signal, $N_{0}$ is the additive white Gaussian noise power spectral density. The integration in (24) can be efficiently computed by Gauss-Hermite quadrature formula (Navidpour et al., 2007; Ghassemlooy et al., 2012b; Alouini and Goldsmith, 1999, Osche, 2002, Yang et al., 2014). Fig. 18 shows the BER plots of OOK-based FSO in atmospheric turbulence corresponding to the open-loop and closed-loop data rate values at various levels of output-error variance. As we can see, the effect of turbulence strength on the amount of signal-to-noise ratio (SNR) is required to maintain a given error performance level. From Fig. 18, it can be inferred that atmospheric turbulence can causes SNR penalty, which might affect the pointing error, for example, around $16 \mathrm{~dB}$ of SNR is needed in open-loop condition to achieve a BER of $10^{-1}$ due to the very weak scintillation of strength $\sigma^{2}=0.0380$. It decreases by over $2 \mathrm{~dB}$ with the closed-loop control as the scintillation strength decreases to $\sigma^{2}=0.0231$, which implies that pointing error control strategy might be required to avoid a BER floor in the system performance. 


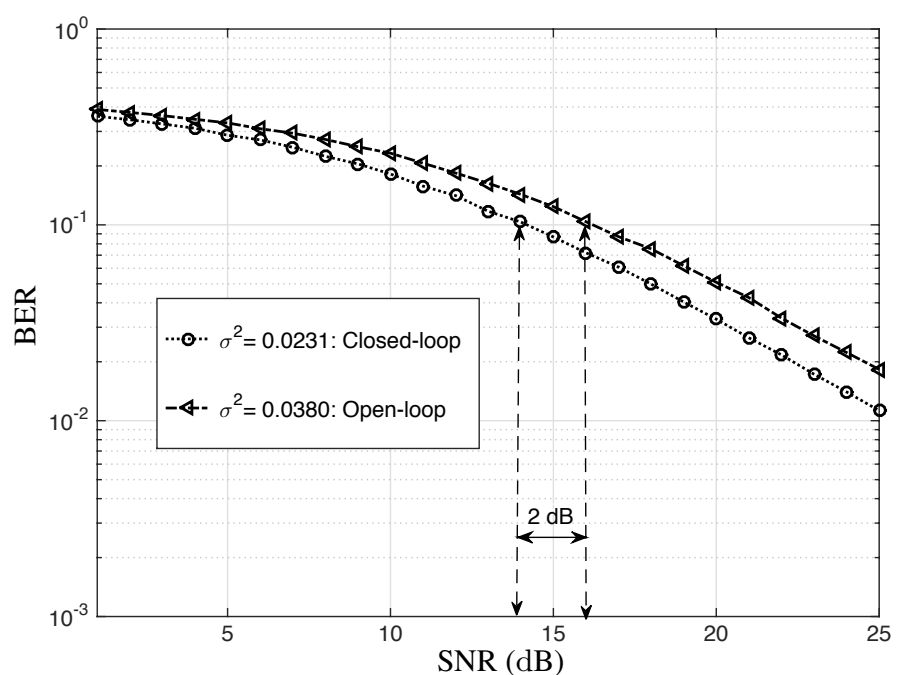

Figure 18: Open-loop and closed-loop BER performances of OOK-based FSO in atmospheric turbulence.

\section{Conclusion}

In this paper, the link performance of the presented FSO link under the influence of the weak atmospheric turbulence has been investigated. The atmospheric turbulence chamber has been characterized theoretically and experimentally for a valid comparison. We found that the fading statistics follow the well-known lognormal distribution that is used for weak turbulence characterization. Based on that, a deterministic nonlinear discrete-time model for pointing error loss due to misalignment has been derived. We then investigate the $\mathcal{H}_{\infty}$ norm optimization problem that guarantees the closed-loop pointing error is stable and ensures the prescribed disturbance attenuation level. The closed-loop pointing error from the numerical simulation shows the center of the optical beam close enough to the receiving aperture center, verifying the efficiency of our proposed robust pointing error control for FSO communication systems.

\section{References}

H. A. Willebrand and B. S. Ghuman. Fiber optics without fiber. IEEE Spectrum, 38:40-45, 2001.

D. Kerr, K. Bouazza-Marouf, K. Girach, and T. C. West. Free space laser communication links for short range control of mobile robots using active pointing and tracking techniques. IEE Colloquium on Optical Free Space Communication Links, pages 11/1-11/5, 1996.

H. M. Oubei, C. Li, K. H. Park, T. Khee, M. S. Alouini, and B. S. Ooi. 2.3 Gbit/s underwater wireless optical communications using directly modulated $520 \mathrm{~nm}$ laser diode. Optics Express, 23(16):20743-20748, 2015.

J. Maynard. System design considerations, chapter Laser Satellite Communication (M. Katzman, ed.), pages 11-67. Prentice-Hall, Berlin, 1987.

V. W. S. Chan. Optical satellite networks. IEEE Journal of Lightwave Technology, 21:2811-2827, 2003.

H. Singh and A. S. Sappal. Moment-based approach for statistical and simulative analysis of turbulent atmospheric channels in FSO communication. IEEE Access, 7:11296-11317, 2019.

H. Henniger and O. Wilfert. An introduction to free-space optical communications. Radio Engineering, 19(1):203-212, 2010.

W. G. Alheadary, K. H. Park, and M. S. Alouini. Performance analysis of multihop heterodyne free-space optical communication over general malaga turbulence channels with pointing error. Optik, 151:34-47, 2015.

E. J. Shin and V. W. S. Chan. Optical communication over the turbulent atmospheric channel using spatial diversity. In IEEE Global Commun. Conf., pages 2055-2060, Taipei, Taiwan, 2002.

A. A. Farid and S. Hranilovic. Outage capacity optimization for free-space optical links with pointing errors. IEEE Journal of Lightwave Technology, 25(7):1702-1710, 2007.

D. K. Borah and D. G. Voelz. Pointing error effects on free-space optical communication links in the presence of atmospheric turbulence. IEEE Journal of Lightwave Technology, 27(18):3965-3973, 2009. 
C. Pontbriand, N. Farr, J. Ware, J. Preisig, and H. Popenoe. Diffuse high-bandwidth optical communications. In OCEANS, pages 1-4, Denver, Colorado, USA, 2008.

I. Rust and H. Asada. A dual-use visible light approach to integrated communication and localization of underwater robots with application to non-destructive nuclear reactor inspection. In IEEE Int. Conf. Robot. Autom., pages 2445-2450, Saint Paul, MN, USA, 2012.

J. Simpson, B. Hughes, and J. Muth. Smart transmitters and receivers for underwater free-space optical communication. IEEE J. Sel. Areas Commun., 30(5):964-974, 2012.

S. Arnon, S. Rotman, and N. S. Kopeika. Beam width and transmitter power adaptive to tracking system performance for free-space optical communication. Applied Optics, 36(24):6095-6101, 2002.

A. Komaee, P. S. Krishnaprasad, and P. Narayan. Active pointing control for short range free-space optical communication. Communications in information and systems, 7(2):177-194, 2007.

H. Yuksel, S. Milner, and C. Davis. Aperture averaging for optimizing receiver design and system performance on free-space optical communication links. Journal of Optical Networking, 4(8):462-475, 2005.

X. Liu. Free-space optics optimization models for building sway and atmospheric interference using variable wavelength. IEEE Transactions on Communications, 57(2):492-498, 2009.

W. Cai, I. N'Doye, B. S. Ooi, M.-S. Alouini, and T.-M. Laleg-Kirati. Modeling and experimental study of the vibration effects in urban free-space optical communication systems. IEEE Photonics Journal, 11(6):7906713, 2019.

W. Cai, I. N'Doye, X. Sun, A. Al-Awan, W. G. Alheadary, M. S. Alouini, B. Ooi, and T. M. Laleg-Kirati. Robust $\mathcal{H}_{\infty}$ pointing error control of free space optical communication systems. In IEEE Conference on Control Technology and Applications (CCTA), Copenhagen, Denmark, 2018.

A. K. Majumdar and J.C. Ricklin. Free-Space Laser Communications: Principles and Advances. Springer, New York, 2008.

X. Zhu and J. M. Kahn. Free-space optical communication through atmospheric turbulence channels. IEEE Trans Communications, 50(8):293-300, 2002.

G. R. Osche. Optical Detection Theory for Laser Applications. NJ: Wiley-Interscience, 1st ed. hoboken edition, 2002.

Z. Ghassemlooy, H. Le Minh, S. Rajbhandari, J. Perez, and M. Ijaz. Performance analysis of ethernet/fast-ethernet free space optical communications in a controlled weak turbulence condition. IEEE Journal of Lightwave Technology, 30 (13):2188-2194, 2012a.

S. Primak, V. Kontorovitch, and V. Lyandres. Stochastic methods and their applications to communications: stochastic differential equations approach. Wiley, 2005.

V. Kontorovich and V. Lyandres. Stochastic differential equations: an approach to the generation of continuous non-Gaussian processes. IEEE Trans. Signal Process., 43(1):2372-2385, 1995.

D. Bykhovsky, D. Elmakayes, and S. Arnon. Experimental evaluation of free space links in the presence of turbulence for server backplane. J. Lightwave Technol., 33:2777-2783, 2015.

A. Neuenkirch and L. Szpruch. First order strong approximations of scalar SDEs defined in a domain. Numerische Mathematik, 128(1):103-136, 2014.

D. Bykhovsky. Free-space optical channel simulator for weak-turbulence conditions. Applied Optics, 54(31):9055-9059, 2015.

Giorgio Volpe and Giovanni Volpe. Numerical simulation of optically trapped particles. In SPIE 9289, 12th Education and Training in Optics and Photonics Conference, volume 9289, page 92890I, Porto, Portugal, 2013.

N. Amari, D. Folio, and A. Ferreira. Robust laser beam tracking control using micro/nano dual-stage manipulators. In IEEE/RSJ International Conference on Intelligent Robots and Systems, pages 1543-1548, Tokyo, Japan, 2013.

N. Amari, D. Folio, and A. Ferreira. Motion of a micro/nano-manipulator using a laser beam tracking system. International Journal of Optomechatronics, 8(1):30-46, 2014.

R. M. Gagliardi and S. Karp. Optical Communication. John Wiley \& Sons, 2nd edition, 1995.

S.P. Boyd, L. El Ghaoui, E. Féron, and V. Balakrishnan. Linear Matrix Inequality in Systems and Control Theory. SIAM, Philadelphia, 1994.

J. Lofberg. YALMIP: a toolbox for modeling and optimization in MATLAB. In IEEE symposium on computer aided control system design, pages $\mathrm{xx}-\mathrm{xx}$, Tapei, Taiwan, 2016.

C. K. Toh, J. M. Todd, and H. R. Tutuncu. SDPT3: a matlab software package for semidefinite programming. Optimization Methods and Software, 11:545-581, 1999. 
M. S. Alouini and M. K. Simon. Dual diversity over correlated log-normal fading channels. IEEE Transactions on Communications, 50:1946-1959, 2002.

Z. Ghassemlooy, W. Popoola, and S. Rajbhandari. Optical Wireless Communications: System and Channel Modelling with MATLAB. CRC Press, Berlin, 1st edition, 2012b.

F. Yang, J. Cheng, and T. A. Tsiftsis. Free-space optical communication with nonzero boresight pointing errors. IEEE Transactions on Communications, 64(2):713-725, 2014.

S. M. Navidpour, M. Uysal, and M. Kavehrad. BER performance of free-space optical transmission with spatial diversity. IEEE Transactions on Communications, 6(8):2813-2819, 2007.

M.S. Alouini and A.J. Goldsmith. A unified approach for calculating error rates of linearly modulated signals over generalized fading channels. IEEE Transactions on Communications, 47(9):1324-1334, 1999. 